\title{
É possível identificar a dengue em crianças a partir do critério de caso suspeito preconizado pelo Ministério da Saúde?
}

\author{
Is it possible to identify dengue in children \\ on the basis of Ministry of Health criteria for suspected dengue cases? \\ Marisa B. P. Rodrigues ${ }^{1}$, Heliane B. M. Freire ${ }^{2}$, Paulo R. L. Corrêa ${ }^{3}$, \\ Marislaine L. Mendonça4, Maria Regina I. Silva5, Elizabeth B. França²
}

\section{Resumo}

Objetivos: Identificar características clínicas indicativas de dengue e avaliar a aplicabilidade, na criança, do critério de caso suspeito do Ministério da Saúde.

Métodos: Estudo transversal, realizado no Centro Geral de Pediatria, Fundação Hospitalar de Minas Gerais. Foram incluídas crianças com doença febril aguda sem etiologia definida, com duração $>24$ horas e $\leq 7$ dias, procedentes da Região Metropolitana de Belo Horizonte. Foram investigadas variáveis clínicas, realizados exames específicos e dosagem de aspartato-aminotransferase, em período considerado endêmico e epidêmico da doença. $O$ grupo de crianças com dengue foi comparado com o grupo com doenças febris agudas inespecíficas. 0 critério de caso suspeito do Ministério da Saúde foi avaliado.

Resultados: $O$ diagnóstico de dengue foi feito em 50,4\% das 117 crianças incluídas no estudo. Não houve associação estatisticamente significativa entre a doença e a maioria dos sintomas analisados. Somente o exantema associou-se mais à dengue (Razão de Prevalência = 1,49; IC a 95\%: 1,05-2,11). A sensibilidade do critério de caso suspeito de dengue foi de somente $50,8 \%$, e o valor preditivo positivo de $62,5 \%$. Esses valores foram maiores nos escolares e no período de maior incidência da doença.

Conclusões: A dengue é freqüente entre as doenças febris na infância, com prevalência variando de acordo com a situação epidemiológica. A clínica das crianças com dengue foi muito semelhante à das crianças com outras doenças inespecíficas. $O$ critério de caso suspeito do Ministério da Saúde mostrou-se de pouca utilidade, principalmente nas crianças menores e em períodos de menor incidência.

J Pediatr (Rio J). 2005;81(3):209-15: Dengue/diagnóstico, febre, sinais e sintomas, criança, pré-escolar, exantema.

\section{Abstract}

Objectives: To identify clinical characteristics indicative of dengue and to evaluate the applicability to children of the Health Ministry criteria for suspected cases.

Methods: A cross-sectional study undertaken at the General Pediatrics Center of the Fundação Hospitalar de Minas Gerais. Children were enrolled if presenting acute febrile conditions with no definite etiology, lasting $>24$ hours and $<7$ days and if resident in the Metropolitan Region of Belo Horizonte. Clinical variables were investigated, specific tests were performed and aspartateaminotransferase assayed, during a period considered both endemic and epidemic for the disease. The subset of children who did have dengue was compared with the subset of nonspecific acute febrile diseases. The Health Ministry criteria for suspected cases was evaluated.

Results: Dengue was diagnosed in $50.4 \%$ of the 117 children studied. There were no statistically significant associations between the disease and the majority of the symptoms analyzed. Only exanthema was more often associated with dengue (Prevalence Ratio $=1.49 ; 95 \%$ CI: $1.05-2.11$ ). The criteria for suspected cases of dengue had a sensitivity of just $50.8 \%$ and a positive predictive value of $62.5 \%$. These values were greater among schoolchildren and during the period of greater disease incidence.

Conclusions: Dengue is common among febrile diseases of childhood, with prevalence that varies according to the epidemiological situation. The clinical status of children with dengue was very similar to that of children with other nonspecific diseases. The Health Ministry criteria for suspected cases were shown to be of little use, particularly with smaller children and during periods of reduced incidence.

J Pediatr (Rio J). 2005;81(3):209-15: Dengue/diagnosis, fever, signs and symptoms, child, preschool, exanthema.

1. Mestre. Programa de Pós-Graduação em Ciências da Saúde, Faculdade de Medicina, Universidade Federal de Minas Gerais (UFMG), Belo Horizonte, MG.

2. Doutora em Medicina, Faculdade de Medicina, UFMG, Belo Horizonte, MG.

3. Mestre em Epidemiologia, Faculdade de Medicina, UFMG, Belo Horizonte, MG

4. Pediatra. Coordenação de Atenção à Criança, Secretaria Municipal de Saúde de Belo Horizonte, MG

5. Pediatra. Gerência de Epidemiologia do Distrito Sanitário Leste, Secretaria Municipal de Saúde de Belo Horizonte, MG.

Fonte financiadora: O presente estudo faz parte do projeto "Reemergência da dengue em Belo Horizonte - Avaliação e Proposta de Intervenção", financiado pelo CNPq (Processo 521158/98-2).

Artigo submetido em 13.10.04, aceito em 22.12.04.

Como citar este artigo: Rodrigues MB, Freire HB, Corrêa PR, Mendonça ML, Silva MR, França EB. É possível identificar a dengue em crianças a partir do critério de caso suspeito preconizado pelo Ministério da Saúde? J Pediatr (Rio J). 2005;81:209-15. 


\section{Introdução}

A dengue é hoje sério problema de saúde pública no Brasil. A infecção pelos vírus da dengue (sorotipos DEN-1, DEN-2, DEN-3 e DEN-4) leva a manifestações clínicas variáveis, podendo ocorrer desde infecção assintomática ou febre indiferenciada até formas graves com hemorragia e/ou choque. Os casos clínicos enquadram-se em três grupos principais: a) dengue clássica; b) febre hemorrágica da dengue/síndrome de choque da dengue - FHD/SCD; c) dengue com complicações ${ }^{1}$.

Segundo o Ministério da Saúde, um caso suspeito de dengue clássico apresenta febre com duração máxima de 7 dias, acompanhada de pelo menos dois dos seguintes sintomas: cefaléia, dor retro-orbitária, mialgia, artralgia, prostração, exantema. Além desses sintomas, deve ter estado nos últimos 15 dias em área onde esteja ocorrendo transmissão de dengue ou tenha a presença de Aedes aegypti.

A Organização Mundial de Saúde (OMS) estima a ocorrência anual de vinte milhões de casos de infecção por dengue. Em muitos países, a dengue acomete principalmente crianças e, na Ásia tropical, é uma das principais causas de hospitalização e morte infantil ${ }^{2}$. No Brasil, a primeira epidemia de dengue documentada clínica e laboratorialmente ocorreu em 1981/82 em Roraima ${ }^{3}$. Em Belo Horizonte, os primeiros casos de dengue foram registrados em 1996. Em 1998, ocorreu epidemia de grande magnitude seguida de período endêmico em 1999 e $2000^{4}$. As taxas de incidência da doença no município sempre foram maiores nos primeiros semestres. Em 2002, de fevereiro a maio, ocorreram mais de 150 casos de dengue por semana, dos quais somente $6 \%$ foram em crianças $^{5}$.

A maioria das infecções por dengue nas crianças é assintomática ou oligossintomática. Estudo na Tailândia demonstrou que $87 \%$ das infecções por dengue resultaram em somente um dia de ausência à escola 6 . Inquérito sorológico realizado no Rio de Janeiro encontrou sorologia positiva em $33 \%$ dos escolares, apesar dos pais informarem que eles não tinham tido a doença7.

$\mathrm{Na}$ infância, muitas vezes é difícil diferenciar a dengue de outras doenças febris agudas. Estudo em crianças tailandesas com rubor facial e febre indiferenciada de curta duração diagnosticou dengue em $35 \%$ das crianças ${ }^{8}$, sendo que, em estudo anterior feito em período epidêmico, essa taxa tinha sido de $90 \%{ }^{9}$. No Paquistão, a freqüência de dengue em crianças com febre de curta duração sem etiologia definida foi de $26 \%{ }^{10}$. Em diversos estudos, os sintomas clássicos de dengue (cefaléia, artralgia, mialgia) foram encontrados com freqüência semelhante nas crianças com dengue e com outras doenças febris ${ }^{11-13}$. Em Cuba, durante o surto epidêmico de 1997, o exantema foi o sinal mais freqüente nas crianças hospitalizadas com dengue ${ }^{14}$.

A propedêutica laboratorial é de suma importância para a confirmação diagnóstica da dengue na criança. Os dois métodos básicos para o diagnóstico laboratorial são a detecção do vírus ou de anticorpos. A detecção do genoma viral através de reação em cadeia da polimerase (PCR), empregando a transcriptase reversa, tem sido utilizada com sucesso em vários laboratórios, mostrando-se útil no diagnóstico da dengue ${ }^{15-17}$. Os testes sorológicos mais utilizados são a inibição de hemaglutinação e a reação imunoenzimática de captura de IgM (ELISA-IgM). Este último é simples, rápido e, na maioria dos casos, a confirmação da dengue pode ser feita com uma única amostra de soro obtida após o sexto dia da doença, desde que associada a quadro clínico e epidemiológico ${ }^{3}$. Considerando a inibição de hemaglutinação como padrão-ouro, a sensibilidade do ELISA-IgM atinge 90 a $97 \%$. Podem ocorrer reações falsopositivas em menos de $2 \%$ dos casos, e algumas reações falso-negativas nas infecções secundárias ${ }^{16}$.

Além dos exames sorológicos e/ou virológicos para confirmação da doença, outros exames inespecíficos têm alto valor preditivo positivo, como o aumento das aminotransferases ${ }^{18-20}$. Para o diagnóstico de FHD, são essenciais as alterações hematológicas de aumento de hematócrito e diminuição de plaquetas.

Frente à situação endêmico-epidêmica da dengue em Belo Horizonte e à inespecificidade dos sintomas na criança, o presente estudo tem como finalidade avaliar a aplicabilidade, na criança, do critério de caso suspeito de dengue preconizado pelo Ministério da Saúde e também identificar sinais e sintomas clínicos que ajudem a diferenciar a dengue de outras doenças febris da infância.

\section{Métodos}

Trata-se de estudo transversal, realizado no ambulatório de pronto-atendimento do Centro Geral de Pediatria (CGP) da Fundação Hospitalar de Minas Gerais (FHEMIG). Foram incluídas crianças de 1 a 12 anos de idade com doença febril aguda sem etiologia definida, com duração maior de 24 horas e menor ou igual a 7 dias, residentes na Região Metropolitana de Belo Horizonte.

O estudo foi realizado em duas etapas: um período considerado epidêmico (20 de março a 19 de julho de 2002) e um período endêmico ( 2 de outubro de 2002 a 31 de janeiro de 2003). Durante parte do dia (4 horas), de segunda a sábado, as crianças atendidas no ambulatório do CGP que se enquadravam no critério de inclusão da pesquisa foram encaminhadas para coleta de dados, realizada por acadêmicos do quinto ano de Medicina treinados que, após entrevista e exame clínico, preenchiam ficha padronizada de investigação. Foram coletadas as seguintes variáveis: duração da febre, presença ou ausência de cefaléia, dor retro-orbitária, mialgia, artralgia, prostração, exantema, diarréia, vômito, rubor facial, irritabilidade, dor de garganta, coriza, tosse, dor abdominal, desidratação, manifestações hemorrágicas, sintomas neurológicos, hepatomegalia. Nas crianças menores, entrevista detalhada buscava qualquer indício da presença dos sintomas. Foi realizado estudo piloto e seus resultados foram utilizados para adequação do questionário e trabalho de campo.

Na consulta inicial, foi solicitada dosagem de aspartato-aminotransferase (AST) e exames específicos para dengue (PCR e IgG das crianças com até 5 dias de febre, IgM e IgG das crianças com mais de 5 dias de febre). Os 
exames laboratoriais foram realizados na Faculdade de Medicina/UFMG: os específicos (sorológicos e PCR), no Núcleo de Pesquisa e Apoio Diagnóstico (NUPAD), e a dosagem de AST, no Laboratório Central. O sangue foi colhido no laboratório do CGP, centrifugado, e o soro encaminhado, juntamente com uma via da ficha de investigação, no prazo máximo de 4 horas, para o NUPAD. 0 método sorológico utilizado foi o Ultramicroelisa (UMELI$\mathrm{SA}$ ) Dengue, desenvolvido pelo Centro de Imunoensaio de Cuba21,22. Esse é um teste imunoenzimático heterogêneo, na sua variante de captura, que utiliza como fase sólida tiras de ultramicroELISA sensibilizadas com anticorpos contra IgM ou IgG humana. A amostra é incubada nas cavidades da tira, e a IgM ou IgG presente na amostra fixa-se aos anticorpos presentes. Adiciona-se antígeno monoclonal de camundongo contra o vírus Dengue conjugado com fosfatase alcalina, que irá unir-se ao complexo da reação anterior, caso a reação seja positiva. Acrescenta-se substrato fluorigênico, e a intensidade da fluorescência permitirá caracterizar a presença de anticorpo específico contra o vírus da dengue. São utilizados controles positivo e negativo. A amostra é considerada positiva acima de 0,300 no UMELISA DENGUE IgM ${ }^{23}$, e acima de 0,150 no UMELISA DENGUE IgG ${ }^{24}$. No teste PCR, os primers utilizados foram os desenvolvidos por Lanciotti et al. ${ }^{15}$, com controles positivos internos. Considerou-se $55 \mathrm{U} / \mathrm{I}$ como o limite superior de normalidade para a $\mathrm{AST}^{23}$.

No retorno do paciente, após o sexto dia de doença, um médico investigava os sinais e sintomas ocorridos durante o período da doença e solicitava sorologia (IgM) nos casos em que esta ainda não havia sido realizada. Se a criança estava no sexto ou sétimo dia da doença no dia da consulta inicial, não era agendado retorno. O intervalo entre o primeiro atendimento e o retorno variou de um a 20 dias (média de 7 dias). Como não foi feito acompanhamento diário dos pacientes, a informação obtida procurava validar a detecção dos sintomas na primeira entrevista e verificar se novos sintomas surgiram no decorrer da doença. Para facilitar o comparecimento aos retornos, foi fornecido valetransporte. Os resultados do exame foram posteriormente comunicados à família por telefone.

Considerou-se como caso de dengue as crianças com PCR e/ou IgM positivos. O grupo de crianças com dengue foi comparado com o grupo com doenças febris agudas inespecíficas. O critério de caso suspeito do Ministério da Saúde foi avaliado, verificando-se sua sensibilidade e seu valor preditivo na faixa etária pediátrica.

Na análise de associação das variáveis com a ocorrência de dengue, utilizou-se a razão de prevalências para estimar a magnitude da associação de alguns sintomas e sinais com a doença. $O$ valor de $p<0,05$ foi considerado como valor limite para significância estatística. Os dados foram analisados utilizando o programa Epi-Info, versão 6.04.

O estudo foi aprovado pelo Comitê de Ética da Universidade Federal de Minas Gerais (UFMG). Foi obtido consentimento esclarecido por escrito dos responsáveis pela criança.

\section{Resultados}

Foram inicialmente selecionadas 125 crianças. Oito crianças foram excluídas: uma não residia na Região Metropolitana de Belo Horizonte, quatro eram menores de 1 ano, uma tinha mais de 7 dias de febre e, em duas, o protocolo não foi seguido. Das 117 crianças do estudo (50 na primeira etapa e 67 na segunda etapa), $59 \%(n=69)$ eram do sexo masculino, $53 \%(n=62)$ tinham de 1 a 4 anos e $47 \%$ $(n=55)$ de 5 a 12 anos.

O diagnóstico de dengue foi feito em $50,4 \%(n=59)$ das crianças incluídas no estudo, sendo $62 \%(n=31)$ na primeira etapa e $41,8 \%(n=28)$ na segunda etapa. Selecionando-se o período de maior incidência (20 de março a 20 de maio de 2002), 64,1\% das crianças febris incluídas no estudo tiveram dengue, e em período de menor incidência (2 de outubro a 2 de dezembro de 2002), 30,2\%. O exame de PCR foi positivo em 35 casos. Foram detectados três sorotipos, sendo $80 \%(n=28)$ DEN-2, 11,4\% $(n=4)$ DEN-1 e $8,6 \%(n=3)$ DEN-3. A IgG, realizada no primeiro atendimento em $83,8 \%(n=98)$ dos casos, foi positiva em 14 crianças. Dessas, oito tiveram diagnóstico de dengue no presente estudo e evoluíram sem complicações. Chamou a atenção o fato de somente duas crianças terem relato anterior de dengue.

Na Tabela 1, são apresentados os principais sintomas e sinais detectados nas crianças com doenças febris agudas no primeiro atendimento. Verificou-se que não houve associação estatisticamente significativa entre a doença e a maioria dos sintomas analisados. Cefaléia, dor retro-orbitária, mialgia e artralgia, sintomas tipicamente atribuídos à dengue, tiveram freqüência semelhante nos dois grupos. Prostração esteve presente em quase $80 \%$ das crianças, independente do diagnóstico. Somente 0 exantema associou-se mais à dengue $(p=0,08)$. A prevalência da dengue foi 1,49 vezes maior nos casos com exantema. Dor de garganta, coriza e tosse, sintomas típicos de doenças respiratórias agudas, também estiveram presentes nas crianças com dengue. Manifestações hemorrágicas foram pouco freqüentes. A prova do laço foi realizada em 74 crianças, sendo positiva em $13,5 \%$ dos casos de dengue e em $5,4 \%$ nas doenças febris inespecíficas. A dosagem de AST foi obtida em 84 casos. A prevalência de dengue foi 1,64 vezes maior (IC a $95 \%$ : $1,04-2,58)$ entre as crianças com AST elevada. Em alguns casos de dengue, a AST atingiu valores superiores a $1.000 \mathrm{U} / \mathrm{l}$.

Foi avaliada a distribuição dos sintomas nos estratos de crianças de 1 a 4 anos de idade e de 5 a 12 anos. Verificou-se que não houve associação estatisticamente significativa entre os sintomas investigados e a dengue. Da mesma forma, ao se avaliar a presença simultânea de dois sintomas considerados no critério de caso suspeito de dengue (cefaléia, dor retro-orbitária, mialgia, artralgia, prostração e exantema), não foi detectada associação estatisticamente significativa.

$\mathrm{Na}$ Tabela 2, são apresentados os resultados relativos às crianças que compareceram ao retorno $(n=99)$, possibilitando avaliação da presença dos sintomas durante o período 
completo da doença. A maioria dos sintomas persistiu sem associação significativa com a dengue. Prostração e cefaléia mantiveram-se como os sintomas mais freqüentes, tanto no grupo de dengue como nas outras doenças febris. A magnitude da associação do exantema com a dengue foi maior $(R P=1,53 ;$ IC a 95\%: 1,07-2,19), com valor de $p=0,06$.

Tabela 1 - Sintomas e sinais, no primeiro atendimento, nos casos de dengue e não-dengue, em crianças de 1 a 12 anos de idade. Belo Horizonte, 2002/2003

\begin{tabular}{|c|c|c|c|c|c|}
\hline Sintomas & $\begin{array}{c}\text { Dengue } \\
n(\%) \\
(n=59)\end{array}$ & $\begin{array}{c}\text { Não-dengue } \\
n(\%) \\
(n=58)\end{array}$ & $\mathbf{R} \mathbf{P} *$ & IC a $95 \%$ & $\mathbf{p}$ \\
\hline Cefaléia & $37(62,7)$ & $36(62,1)$ & 1,01 & $(0,70-1,47)$ & 0,91 \\
\hline Dor retro-orbitária & $17(29,3)$ & $11(19,0)$ & 1,30 & $(0,90-1,89)$ & 0,28 \\
\hline Mialgia & $23(39,7)$ & $18(31,0)$ & 1,20 & $(0,84-1,73)$ & 0,44 \\
\hline Artralgia & $11(19,0)$ & $13(22,4)$ & 0,90 & $(0,53-1,45)$ & 0,82 \\
\hline Prostração & $47(79,7)$ & $46(79,3)$ & 1,01 & $(0,65-1,58)$ & 0,86 \\
\hline Exantema & $17(28,8)$ & $8(13,8)$ & 1,49 & $(1,05-2,11)$ & 0,08 \\
\hline Diarréia & $10(16,9)$ & $11(19,0)$ & 0,93 & $(0,57-1,52)$ & 0,97 \\
\hline Vômito & $35(59,3)$ & $28(48,3)$ & 1,25 & $(0,89-1,81)$ & 0,31 \\
\hline Epistaxe & $7(11,9)$ & $9(15,8)$ & 0,84 & $(0,47-1,51)$ & 0,73 \\
\hline Rubor facial & $22(37,3)$ & $19(33,3)$ & 1,09 & $(0,75-1,57)$ & 0,80 \\
\hline Irritabilidade & $20(33,9)$ & $11(19,3)$ & 1,41 & $(0,99-1,99)$ & 0,11 \\
\hline Dor de garganta & $14(23,7)$ & $18(31,6)$ & 0,82 & $(0,53-1,27)$ & 0,46 \\
\hline Coriza & $22(37,3)$ & $28(49,1)$ & 0,78 & $(0,54-1,15)$ & 0,27 \\
\hline Tosse & $28(47,5)$ & $29(50,9)$ & 0,93 & $(0,65-1,34)$ & 0,86 \\
\hline Dor abdominal & $14(23,7)$ & $7(12,1)$ & 1,42 & $(0,98-2,06)$ & 0,16 \\
\hline Gengivorragia & $2(3,4)$ & $1(1,7)$ & 1,33 & $(0,59-3,03)$ & 1,00 \\
\hline Petéquias & $2(3,4)$ & $3(5,2)$ & 0,79 & $(0,26-2,33)$ & 0,68 \\
\hline Sangramento intestinal & $2(3,4)$ & $2(3,4)$ & 0,99 & $(0,37-2,69)$ & 1,00 \\
\hline Desidratação & $2(3,4)$ & $5(8,8)$ & 0,55 & $(0,17-1,79)$ & 0,27 \\
\hline Sintomas neurológicos & $1(1,7)$ & $2(3,4)$ & 0,66 & $(0,13-3,28)$ & 0,62 \\
\hline Hepatomegalia & $1(1,7)$ & $2(3,4)$ & 0,66 & $(0,13-3,28)$ & 0,62 \\
\hline
\end{tabular}

* $\mathrm{RP}=$ razão de prevalências.

Tabela 2 - Sintomas e sinais nos casos de dengue e não-dengue nas crianças que compareceram ao retorno (após o sexto dia de doença). Belo Horizonte, 2002/2003

\begin{tabular}{lccccc}
\hline Sintomas & $\begin{array}{c}\text { Dengue } \\
\mathbf{n}(\mathbf{\%}) \\
(\mathbf{n}=\mathbf{5 1})\end{array}$ & $\begin{array}{c}\text { Não-dengue } \\
\mathbf{n}(\mathbf{\%}) \\
\mathbf{( n = 4 8 )}\end{array}$ & $\mathbf{R P} *$ & IC a 95\% & $\mathbf{p}$ \\
\hline Cefaléia & $36(70,6)$ & $30(62,5)$ & 1,20 & $(0,78-1,85)$ & 0,52 \\
Dor retro-orbitária & $16(31,4)$ & $11(22,9)$ & 1,22 & $(0,82-1,81)$ & 0,47 \\
Mialgia & $22(43,1)$ & $18(37,5)$ & 1,12 & $(0,76-1,64)$ & 0,71 \\
Artralgia & $11(21,6)$ & $11(22,9)$ & 0,96 & $(0,60-1,54)$ & 0,94 \\
Prostração & $42(82,4)$ & $40(83,3)$ & 0,97 & $(0,59-1,59)$ & 0,89 \\
Exantema & $18(35,3)$ & $8(16,7)$ & 1,53 & $(1,07-2,19)$ & 0,06 \\
Diarréia & $11(21,6)$ & $9(18,8)$ & 1,09 & $(0,69-1,71)$ & 0,92 \\
Vômito & $33(64,7)$ & $24(50,0)$ & 1,35 & $(0,89-2,04)$ & 0,20 \\
Rubor facial & $18(35,3)$ & $18(37,5)$ & 0,95 & $(0,64-1,43)$ & 0,98 \\
Irritabilidade & $17(33,3)$ & $9(18,8)$ & 1,40 & $(0,97-2,04)$ & 0,16 \\
Dor de garganta & $16(31,4)$ & $14(19,2)$ & 1,05 & $(0,70-1,58)$ & 0,98 \\
Coriza & $24(47,1)$ & $23(47,9)$ & 0,98 & $(0,67-1,44)$ & 0,91 \\
Tosse & $27(52,9)$ & $28(58,3)$ & 0,90 & $(0,61-1,32)$ & 0,74 \\
Dor abdominal & $21(41,2)$ & $14(29,2)$ & 1,28 & $(0,88-1,86)$ & 0,30 \\
Petéquias & $3(5,9)$ & $2(4,2)$ & 1,18 & $(0,56-2,47)$ & 0,94 \\
\hline
\end{tabular}

* RP = razão de prevalências. 
As crianças com e sem dengue foram comparáveis em relação à faixa etária. A mediana da idade nos dois grupos foi de 4 anos, e o teste de Kruskal-Wallis não foi significativo $(p=0,10)$.

Ao classificar as crianças de acordo com o critério de caso suspeito do Ministério da Saúde, verificou-se associação significativa entre ter a doença e se enquadrar no critério $(p=0,05)$, com uma prevalência 1,49 vezes maior (IC a 95\%: 1,04-2,12). Porém, se o diagnóstico fosse baseado nesse critério, 49,2\% das crianças com dengue não teriam sido identificadas. Nas crianças de 1 a 4 anos, a associação não foi estatisticamente significativa. Já na faixa de 5 a 12 anos, a associação entre ter a doença e se enquadrar no critério foi significativa $(p=0,04)$. Entretanto, também nessa faixa etária, se o diagnóstico fosse baseado no critério de caso suspeito do Ministério da Saúde, $33,3 \%$ das crianças com dengue não teriam sido identificadas (Tabela 3).

Foi avaliada a aplicabilidade do critério de caso suspeito nas diferentes faixas etárias e períodos de ocorrência. A sensibilidade do critério de caso suspeito de dengue foi de $50,8 \%$, e a especificidade e valor preditivo positivo de 69 e $62,5 \%$, respectivamente. A sensibilidade e o valor preditivo positivo foram melhores nos escolares e no período de maior incidência da doença (Tabela 4).

\section{Discussão}

Os resultados deste estudo indicam que a dengue é freqüente entre as doenças febris agudas na infância, e que essa prevalência varia de acordo com a situação epidemiológica. Verificou-se maior prevalência no período no qual a Secretaria de Saúde Municipal registrou mais de 200 casos semanais de dengue, indicando ser este um período epidêmico. É provável que exista uma subnotificação importante. Além disso, a dengue na criança é muitas vezes assintomática 7,24 , como evidencia o fato da maior parte das crianças com IgG positiva no primeiro atendimento não terem relato anterior de dengue.

Neste estudo, o quadro clínico das crianças com diagnóstico de dengue foi muito semelhante ao das crianças com outras doenças febris agudas inespecíficas. Estudo anterior mostrou que não houve diferença significativa na freqüência de cefaléia, dor abdominal e manifestações hemorrágicas entre as crianças com dengue e com outras doenças febris acompanhadas de rubor facial, mas que as crianças com dengue apresentaram mais frequentemente anorexia, náusea e vômito e maior positividade da prova do laço, plaquetopenia, leucopenia e elevação de $A S T$ e $A_{L}{ }^{8}$. Outro estudo confirmou que o conjunto de sintomas clássicos de dengue (cefaléia, artralgia, mialgia, exantema, prostração) foi pouco ob-

Tabela 3 - Diagnóstico e inclusão no critério de caso suspeito, por faixa etária, nas crianças com doenças febris agudas. Belo Horizonte, 2002/2003

\begin{tabular}{|c|c|c|c|c|c|c|c|}
\hline \multirow{3}{*}{$\begin{array}{l}\text { Idade } \\
\text { (anos) }\end{array}$} & \multicolumn{4}{|c|}{ Inclusão no critério de caso suspeito } & \multirow{3}{*}{$\mathbf{R P} *$} & \multirow{3}{*}{ IC a $95 \%$} & \multirow{3}{*}{$\mathbf{p}$} \\
\hline & \multicolumn{2}{|c|}{ Sim } & \multicolumn{2}{|c|}{ Não } & & & \\
\hline & Dengue & Não dengue & Dengue & Não dengue & & & \\
\hline 1 a 4 & 12 & 8 & 20 & 22 & 1,26 & $0,78-2,03$ & 0,52 \\
\hline 5 a 12 & 18 & 10 & 9 & 18 & 1,93 & $1,06-3,52$ & 0,04 \\
\hline Total & 30 & 18 & 29 & 40 & 1,49 & $1,04-2,12$ & 0,05 \\
\hline
\end{tabular}

* $\mathrm{RP}=$ razão de prevalências.

Tabela 4 - Validade do critério de caso suspeito de dengue em diferentes faixas etárias e períodos de ocorrência. Belo Horizonte, 2002/2003

\begin{tabular}{|c|c|c|}
\hline Variáveis & $\begin{array}{c}\text { Sensibilidade } \\
(\%)\end{array}$ & $\begin{array}{c}\text { VPP } * \\
(\%)\end{array}$ \\
\hline \multicolumn{3}{|l|}{ Idade da criança (anos) } \\
\hline 1 a 12 & 50,8 & 62,5 \\
\hline 1 a 4 & 37,5 & 60,0 \\
\hline 5 a 12 & 66,7 & 64,3 \\
\hline \multicolumn{3}{|l|}{ Períodos } \\
\hline Período inicial da $1^{\mathrm{a}}$ etapa ${ }^{\dagger}$ & 68,0 & 89,5 \\
\hline Período inicial da $2^{\mathrm{a}}$ etapa ${ }^{\neq}$ & 15,4 & 16,7 \\
\hline
\end{tabular}

* VPP = valor preditivo positivo.

† Correspondente a 20 de março/2002 a 20 de maio/2002.

‡ Correspondente a 02 de outubro/2002 a 02 de dezembro/2002. 
servado nas crianças, e a freqüência foi semelhante nos casos de dengue e outras doenças febris; cefaléia, coriza, tosse e diarréia foram significativamente mais freqüentes nas doenças febris inespecíficas enquanto o exantema foi mais comum na dengue ${ }^{11}$. Comparação similar foi realizada no Vietnã: sintomas classicamente atribuídos à dengue tiveram freqüência semelhante nos dois grupos, e o grupo com dengue apresentou menos sintomas respiratórios e mais sintomas gastrointestinais ${ }^{13}$.

No presente estudo, somente o exantema apresentou associação próxima ao limite de significância. Revisão feita em Uberlândia, apesar de englobar todas as faixas etárias, corrobora esse achado ${ }^{12}$. Considerando os sintomas relatados nas crianças que compareceram aos retornos, a magnitude dessa associação tornou-se maior, evidenciando que, em muitos casos, o exantema não se manifesta nos primeiros dias. Deve-se ressaltar, entretanto, que resultados não significativos observados podem ser devidos ao pequeno tamanho da amostra. A elevação da AST foi mais freqüente nas crianças com dengue, atingindo níveis muito elevados em alguns casos, o que reforça a importância do comprometimento hepático na doença $18,19,25$. Não houve diferença na ocorrência de dengue e outras doenças febris em relação às faixas etárias, o que difere do trabalho de Kalayanarooj et al., na Tailândia, que evidenciou maior freqüência de dengue nas crianças mais velhas ${ }^{8}$. Verificouse que, mesmo nessas, o quadro clínico da dengue é muito semelhante ao de outras doenças febris inespecíficas, o que difere da crença de que nos escolares os sintomas seriam semelhantes aos dos adultos 2,16 .

Na presente casuística, nenhum caso se enquadrou nos critérios de FHD, e somente oito crianças tiveram infecção secundária. Entretanto, a hemoconcentração e a trombocitopenia não puderam ser avaliadas adequadamente, porque o hemograma deixou de ser solicitado em algumas crianças. Manifestações hemorrágicas, hepatomegalia e prova do laço positiva foram pouco freqüentes e sem diferença significativa entre os dois grupos.

Neste estudo, o diagnóstico de dengue foi considerado nos casos com PCR e/ou IgM positivas. Os casos com IgM positiva em amostra única seriam casos "prováveis" de dengue, uma vez que esse achado pode persistir por até 90 dias. Apesar disso, casos "prováveis" e casos "confirmados" têm sido analisados conjuntamente em alguns trabaIhos $^{14,26,27}$. O Ministério da Saúde considera caso confirmado de dengue aquele com IgM positiva associada a quadro clínico e epidemiológico ${ }^{3}$. Verificou-se, assim, que cerca de $50 \%$ das crianças com doenças febris agudas inespecíficas tinham dengue. O critério de caso suspeito do Ministério da Saúde mostrou-se de pouca utilidade, principalmente nas crianças menores de 5 anos e fora de períodos epidêmicos. Se o diagnóstico fosse baseado nesse critério, quase metade das crianças com dengue não teriam sido identificadas. A maior dificuldade de diagnóstico em período não-epidêmico já tinha sido relatada em estudo anterior ${ }^{28}$.

Chamou atenção a detecção de circulação simultânea de três sorotipos, situação epidemiológica de risco para o surgimento de FHD. Esse fato reforça a necessidade de vigilância e busca ativa de casos, de modo a possibilitar a identificação oportuna da doença. Uma das medidas preconizadas pela OMS é a vigilância da febre ${ }^{2}$, onde clínicas sentinelas notificam à autoridade sanitária semanalmente 0 número de pacientes febris atendidos, para a detecção de aumentos anormais de doenças febris. Outra estratégia seria a realização de exames específicos para dengue nas amostras sorológicas coletadas rotineiramente nos programas de erradicação das doenças exantemáticas e também, periodicamente, em amostragem de crianças com doenças febris agudas inespecíficas.

Em síntese, os resultados deste estudo indicam que é muito difícil diferenciar clinicamente a dengue de outras doenças febris da infância, não se devendo dissociar a clínica da epidemiologia. O apoio do laboratório torna-se essencial nessa faixa etária, principalmente nos períodos de baixa incidência. O profissional de saúde deve estar sempre informado da situação epidemiológica da doença, ficando alerta diante de quadros febris agudos de etiologia indefinida, para diagnóstico precoce e acompanhamento adequado.

\section{Agradecimentos}

Os autores agradecem aos médicos do Centro Geral de Pediatria, à Dra. Maria Aparecida Martins, pela contribuição na fase de elaboração do projeto e às Dras. Talitah Candiani e Letícia Lacerda, que participaram da coleta de dados.

\section{Referências}

1. Ministério da Saúde. Fundação Nacional de Saúde (FUNASA). Dengue: diagnóstico e manejo clínico. Brasília; Fundação Nacional de Saúde; 2002. 28p.

2. Organização Mundial de Saúde. Dengue hemorrágica: diagnóstico, tratamento, prevenção e controle. $2^{a}$ ed. São Paulo; 2001. 84p.

3. Ministério da Saúde. Fundação Nacional de Saúde (FUNASA). Departamento de Operações. Coordenação de Controle de Doenças Transmitidas por Vetores. Manual de dengue - vigilância epidemiológica e atenção ao doente. $2^{\mathrm{a}}$ ed. Brasília; DEOPE; 1996. 79p.

4. Corrêa PRL, França E. Reemergência do dengue em Belo Horizonte: (I) surgimento de epidemias entre 1996 e 2000. Rev Bras Epidemiol. 2002; Supl Es:240.

5. Secretaria Municipal de Saúde de Belo Horizonte. Dados da dengue em BH [site na Internet]. Disponível: www.pbh.gov.br/ bhdengue.

6. Burke DS, Nisalak A, Johnson DE, Scott RM. A prospective study of dengue infections in Bangkok. Am J Trop Hyg. 1988;38:172-80.

7. Figueiredo LT, Cavalcante SM, Simões MC. Encuesta serológica sobre el dengue entre escolares de Rio de Janeiro, Brasil, 1986 y 1987. Bol Oficina Sanit Panam. 1991;111:525-33.

8. Kalayanarooj S, Vaughn DW, Nimmannitya S, Green $S$, Suntayakorn S, Kunentrasai N, et al. Early clinical and laboratory indicators of acute dengue illness. J Infect Dis. 1997;176:313-21.

9. Teeraratkul A, Limpakarnjanara K, Nisalak A, Nimmannitya S. Predictive value of clinical and laboratory findings for early diagnosis of dengue and dengue hemorrhagic fever. Southeast Asian J Trop Med Public Health. 1990;21:696-7.

10. Akram DS, Igarashi A, Takasu T. Dengue virus infection among children with undifferentiated fever in Karachi. Indian J Pediatr. 1998;65:735-40. 
11. Endy TP, Chunsuttiwat S, Nisalak A, Libraty DH, Green S, Rothman $A L$, et al. Epidemiology of inapparent and symptomatic acute dengue virus infection: a prospective study of school children in Kamphaeng Phet, Thailand. Am J Epidemiol. 2002;156:40-51.

12. Nunes-Araújo FR, Ferreira MS, Nishioka A. Dengue fever in Brazilian adults and children: assessment of clinical findings and their validity for diagnosis. Ann Trop Med Parasitol. 2003;97: 415-9.

13. Phuong CX, Nhan NT, Kneen R, Thuy PT, Thien CV, Nga NT, et al. Clinical diagnosis and assessment of severity of confirmed dengue infections in Vietnamese children: is the world health organization classification helpful? Am J Trop Med Hyg. 2004;70:172-9.

14. Caballero MEV, Portuondo TMA, Serrano HP. Características clínicas de la febre de dengue em niños durante el brote epidémico en Santiago de Cuba. Rev Cuba Med Trop. 2001;53: 20-3.

15. Lanciotti RS, Calisher CH, Gubler DJ, Chang GJ, Vorndam AV. Rapid detection and typing of dengue viruses from clinical samples by using reverse transcriptase-polymerase chain reaction. J Clin Microbiol. 1992;30:545-51.

16. Guzmán MG, Kouri G. Dengue: an update. Lancet Infect Dis. 2002;2:33-42.

17. Sa-ngasang A, Wibulwattanakij $S$, Chanama $S$, O-rapinpatipat A, A-nuegoonpipat A, Anantapreecha S, et al. Evaluation of RT$\mathrm{PCR}$ as a tool for diagnosis of secondary dengue virus infection. Jpn J Infect Dis. 2003;56:205-9.

18. Dietz V, Gubler DJ, Ortiz S, Kuno G, Casta-Vélez A, Sather GE, et al. The 1986 dengue and dengue hemorrhagic fever epidemic in Puerto Rico: epidemiologic and clinical observations. PR Health Sci J. 1996;15:201-10.

19. Wahid SF, Sanusi S, Zawawi MM, Ali RA. A comparison of the pattern of liver involvement in dengue hemorrhagic fever with classic dengue fever. Southeast Asian J Trop Med Public Health. 2000;31:259-63.

20. Shivbalan S, Anandnathan K, Balasubramanian S, Datta M, Amalraj E. Predictors of spontaneous bleeding in dengue. Indian J Pediatr. 2004;71:33-6.
21. Garcia S, Herrera R, Solís R, Jaramillo A, Berro O, Urquiza HD. Evaluación del desepeño del UMELISA dengue IgM en el diagnóstico del dengue. Centro de Inmunoensayo, Cuba; Escuela Colombiana de Medicina, Colômbia; Laboratório Central Instituto Noel Nutels, Brasil. [1996]. Relatório.

22. Mexico. Instituto Mexicano Del Seguro Social. Evaluación de reactivos UMELISA para detección de anticorpos IgM específicos contra dengue. México: Direccion de prestaciones medicas Coordenacion de Salud Comunitária; 1998.

23. UMELISA DENGUE IgM. Centro de Immunoensayo/Instituto Pedro Kourí, Cuba 1995. Bula de teste.

24. UMELISA DENGUE IgG. Centro de Immunoensayo/Instituto Pedro Kourí, Cuba 1995. Bula de teste.

25. Nicholson JK, Pesce MA. Faixas de referências para exames laboratoriais e procedimentos. In: Behrman RE, Kliegman RM, Jenson HB. Nelson Tratado de Pediatria. $16^{\mathrm{a}}$ ed. Rio de Janeiro: Editora Guanabara Koogan; 2002.

26. Graham RR, Jiffrie M, Tan R, Hayes CG, Laksono I, Maroef C, et al. A prospective seroepidemiologic study on dengue in children four to nine years of age in Yogyakarta, Indonesia. Am J Trop Hyg. 1999;61:412-9.

27. Mohan B, Patwari AK, Anand VK. Hepatic dysfunction in childhood dengue infection. J Trop Pediatr. 2000;46:40-3.

28. Rigau-Pérez JG, Ayala-López A, Vorndam AV, Clark GG. Dengue activity in Puerto Rico during an interepidemic period (199597). Am J Trop Med Hyg. 2001;64:75-83.

\section{Correspondência:}

Marisa Bicalho Pinto Rodrigues

Rua Carlos Gomes, 83/302

CEP 30350-130 - Belo Horizonte, MG

Tel.: (31) 3296.4923/3335.8835

Fax: (31) 3335.8835

E-mail: marisabpr@uol.com.br 